\title{
ORIENTATION-PRESERVING SELF-HOMEOMORPHISMS OF THE SURFACE OF GENUS TWO HAVE POINTS OF PERIOD AT MOST TWO
}

\author{
WARREN DICKS AND JAUME LLIBRE \\ (Communicated by Mary Rees)
}

\begin{abstract}
We show that for any orientation-preserving self-homeomorphism $\alpha$ of the double torus $\Sigma_{2}$ there exists a point $p$ of $\Sigma_{2}$ such that $\alpha(\alpha(p))=p$. This answers a question raised by Jakob Nielsen in 1942 .
\end{abstract}

\section{BACKGROUND}

Throughout this article, $R$ will denote a commutative ring, and $g$ a positive integer. We shall write $\Sigma_{g}$ to denote the closed, connected, orientable surface of genus $g$, and $\mathbb{Z}_{g}$ to denote the ring $\mathbb{Z} / g \mathbb{Z}$.

Nielsen [10] (cf. [7]) showed that, for any $g \geq 2$, there exists an orientationpreserving self-homeomorphism $\alpha$ of $\Sigma_{g}$ such that $\alpha, \alpha^{2}, \ldots, \alpha^{2 g-3}$ are all fixedpoint free, that is, have no fixed-points. He showed further that, for any orientationpreserving self-homeomorphism $\alpha$ of $\Sigma_{g}$, at least one of $\alpha, \alpha^{2}, \ldots, \alpha^{2 g-3}, \alpha^{2 g-2}$ has a fixed-point if $g \geq 3$, and at least one of $\alpha, \alpha^{2}, \alpha^{3}$ has a fixed-point if $g=2$.

Since $2 g-2=2$ for $g=2$, this left open the question of exactly what happens in the case $g=2$, and he commented that it seemed difficult to him [10, Section 4] (cf. [7]). The problem has not been forgotten; for example, in a recent paper in which he obtains results analogous to Nielsen's for orientation-reversing selfhomeomorphisms and for non-orientable surfaces, Wang [13] mentions that it is still open. In the next section we solve this problem, rounding off Nielsen's abovementioned results by showing that, for $g=2$, at least one of $\alpha, \alpha^{2}$ has a fixed-point, or, equivalently, $\alpha^{2}$ has a fixed-point.

This result has its origins in classical topology, has connections with dynamical systems, and has a proof which is mainly algebraic. Let us review the basic information that will be used.

Recall that the fundamental group of $\Sigma_{g}$ has a one-relator presentation

$$
\pi_{1}\left(\Sigma_{g}\right)=\left\langle x_{i}, y_{i}(1 \leq i \leq g) \mid \prod_{i=1}^{g}\left[x_{i}, y_{i}\right]=1\right\rangle,
$$

where $[x, y]$ denotes $x y x^{-1} y^{-1}$. Recall also that the first homology $R$-module of $\Sigma_{g}$, denoted $\mathrm{H}_{1}\left(\Sigma_{g}, R\right)$, is the $R$-module obtained by abelianizing the fundamental group and then tensoring over $\mathbb{Z}$ with $R$. There is a natural group homomorphism

Received by the editors September 22, 1994.

1991 Mathematics Subject Classification. Primary 55M20; Secondary 57M20, 54H20, 57M60. 
$\pi_{1}\left(\Sigma_{g}\right) \rightarrow \mathrm{H}_{1}\left(\Sigma_{g}, R\right)$, which we shall denote $x \mapsto[x]$. Thus $\mathrm{H}_{1}\left(\Sigma_{g}, R\right)$ is the free $R$ module with basis $\left[x_{i}\right],\left[y_{i}\right](1 \leq i \leq g)$. Moreover the algebraic intersection number determines a symplectic form on $\mathrm{H}_{1}\left(\Sigma_{g}, R\right)$, namely, the anti-symmetric bilinear form

$$
\mathrm{H}_{1}\left(\Sigma_{g}, R\right) \times \mathrm{H}_{1}\left(\Sigma_{g}, R\right) \rightarrow R,(x, y) \mapsto\langle x, y\rangle,
$$

such that

$$
\left\langle\left[x_{i}\right],\left[x_{j}\right]\right\rangle=\left\langle\left[y_{i}\right],\left[y_{j}\right]\right\rangle=0,\left\langle\left[x_{i}\right],\left[y_{j}\right]\right\rangle=-\left\langle\left[y_{j}\right],\left[x_{i}\right]\right\rangle=\delta_{i, j}(1 \leq i, j \leq g) ;
$$

for details, see, for example, [4].

Now let $\alpha: \Sigma_{g} \rightarrow \Sigma_{g}$ be a continuous self-map. Any choice of a point $p$ of $\Sigma_{g}$, and a homotopy class $x$ of paths in $\Sigma_{g}$ from $p$ to $\alpha(p)$, determines an endomorphism $\pi_{1}(\alpha)$ of $\pi_{1}\left(\Sigma_{g}, p\right)$, defined by $y \mapsto x \cdot \alpha(y) \cdot x^{-1}$. This group endomorphism $\pi_{1}(\alpha)$ need not be uniquely determined by $\alpha$, but it is unique up to composition with an inner automorphism. Hence $\pi_{1}(\alpha)$ determines an $R$-module endomorphism $\mathrm{H}_{1}(\alpha, R)$ of $\mathrm{H}_{1}\left(\Sigma_{g}, R\right)$, which is uniquely determined by $\alpha$, and it respects the symplectic structure; for details, see, for example, [4].

Let $A=\mathrm{H}_{1}(\alpha, R)$. Since we have a specific $R$-basis of $\mathrm{H}_{1}\left(\Sigma_{g}, R\right)$, we can represent $A$ by a $2 g \times 2 g$ matrix over $R$. This matrix has a characteristic polynomial (resp. a determinant, a trace) denoted $\chi(A)(t)=\operatorname{det}\left(t I_{2 g}-A\right) \in R[t]($ resp. $\operatorname{det}(A)$, $\operatorname{trace}(A))$. This will be denoted $\chi_{R}(\alpha)(t)\left(\operatorname{resp} \cdot \operatorname{det}_{R}(\alpha), \operatorname{trace}_{R}(\alpha)\right)$.

Suppose that $\alpha$ is an orientation-preserving self-homeomorphism of $\Sigma_{g}$. Then $\operatorname{det}_{R}(\alpha)=1$; for details, see, for example, [11, II.5]. By the Lefschetz fixed-point theorem for compact polyhedra and by Poincaré duality for surfaces, if $\alpha$ is also fixed-point free, then trace ${ }_{\mathbb{Z}}(\alpha)=2$; for details, see, for example, [12], [3]. Further, it is easy to see that if $\operatorname{trace}_{\mathbb{Z}}(\alpha)=2$, then $\operatorname{trace}_{R}(\alpha)=2$, and it is this consequence which interests us.

\section{Results}

We begin with a ring-theoretic fact that we shall need.

2.1 Lemma. There exists a commutative ring $R$ such that $7 R=R, R$ has an element $r$ such that $\sum_{i=0}^{6} r^{i}=0$, and for every $B \in \mathrm{GL}_{2}(R)$, $\operatorname{trace}\left(B^{t} B^{-1}\right) \neq 0$, where $t$ denotes the transpose.

Proof. Set $R=\mathbb{Z}_{4}[t] /\left(t^{3}+2 t^{2}+t-1\right)$. As $\mathbb{Z}_{4}$-module, $R$ is free of rank three, so $2 R \neq 0$.

Also $R / 2 R \approx \mathbb{Z}_{2}[t] /\left(t^{3}+t+1\right)$, a field of eight elements, so 2 is not a unit in $R$ and $2 R$ is a maximal ideal of $R$.

Notice that $7 R=-R=R$.

Let $r$ denote the class of $t$ in $R$. Then $r^{3}+2 r^{2}+r-1=0$, so

$$
\sum_{i=0}^{6} r^{i}=4 r+\left(r^{3}+2 r^{2}+r-1\right)\left(r^{3}-r^{2}+2 r-1\right)=0+0=0 .
$$

Now suppose that there exists $B \in \mathrm{GL}_{2}(R)$ with $\operatorname{trace}\left(B^{t} B^{-1}\right)=0$. Say $B=$ $\left(\begin{array}{ll}a & b \\ c & d\end{array}\right)$. Write $u=a d-b c$ and $v=b+c$. Now $u$ is a unit of $R$ and

$$
B^{t} B^{-1}=\left(\begin{array}{ll}
a & c \\
b & d
\end{array}\right)\left(\begin{array}{cc}
d & -b \\
-c & a
\end{array}\right) u^{-1}=\left(\begin{array}{ll}
a d-c^{2} & -a b+c a \\
b d-d c & -b^{2}+a d
\end{array}\right) u^{-1} .
$$


So we have $0=\operatorname{trace}\left(B^{t} B^{-1}\right)=\left(a d-c^{2}-b^{2}+a d\right) u^{-1}$. Thus $b^{2}+c^{2}=-2 a d$, so

$$
v^{2}=(b+c)^{2}=b^{2}+2 b c+c^{2}=b^{2}+c^{2}+2 b c=-2 a d+2 b c=-2 u .
$$

Since 2 is not a unit in $R$, we see that $v$ is not a unit in $R$, so

$$
R \supset v R \supseteq(v R)^{2}=v^{2} R=-2 u R=2 R .
$$

By the maximality of $2 R$, we see that $v R=2 R$, and on squaring we see that $v^{2} R=4 R$. Hence $2 R=v^{2} R=4 R=0$, a contradiction, as desired.

We next recall a result from [10, Section 4] (cf. [7]).

2.2 Lemma (Nielsen). If $\alpha$ is an orientation-preserving self-homeomorphism of $\Sigma_{2}$ such that $\alpha^{2}$ is fixed-point free, then, for any commutative ring $R$,

$$
\chi_{R}(\alpha)(t)=t^{4}-2 t^{3}+t^{2}-2 t+1
$$

in $R[t]$.

Proof. Without loss of generality, we take $R=\mathbb{Z}$.

By the hypotheses, $\operatorname{det}_{\mathbb{Z}}(\alpha)=1$ and, since $\alpha^{2}, \alpha$, and $\alpha^{-1}$ are fixed-point free, by the Lefschetz fixed-point theorem, $\operatorname{trace}_{\mathbb{Z}}\left(\alpha^{2}\right)=\operatorname{trace}_{\mathbb{Z}}(\alpha)=\operatorname{trace}_{\mathbb{Z}}\left(\alpha^{-1}\right)=2$. Let $A=\mathrm{H}_{1}(\alpha, \mathbb{Z})$. Hence $\operatorname{det}(A)=1$ and $\operatorname{trace}\left(A^{2}\right)=\operatorname{trace}(A)=\operatorname{trace}\left(A^{-1}\right)=2$. Also $\chi(A)(t)=\chi_{\mathbb{Z}}(\alpha)(t)$.

Say $\chi(A)(t)=t^{4}-s_{1} t^{3}+s_{2} t^{2}-s_{3} t+s_{4}$. Using certain well-known identities, which are easily verified for upper triangular $4 \times 4$ complex matrices, and hence true for all $4 \times 4$ complex matrices, we have

$$
\begin{aligned}
& s_{1}=\operatorname{trace}(A)=2, \\
& s_{2}=\frac{1}{2}\left((\operatorname{trace}(A))^{2}-\operatorname{trace}\left(A^{2}\right)\right)=\frac{1}{2}\left(2^{2}-2\right)=1, \\
& s_{3}=\operatorname{trace}\left(A^{-1}\right) \operatorname{det}(A)=2, \\
& s_{4}=\operatorname{det}(A)=1 . \square
\end{aligned}
$$

We now arrive at our main result.

2.3 Theorem. If $\alpha$ is an orientation-preserving self-homeomorphism of $\Sigma_{2}$, then $\alpha^{2}$ has a fixed-point.

Proof. Suppose not, so $\alpha^{2}$ is fixed-point free. We proceed to derive a contradiction.

Let $1_{\Sigma_{2}}$ denote the identity map on $\Sigma_{2}$. For any $t \in \mathbb{Z}, t \mathrm{H}_{1}\left(1_{\Sigma_{2}}, \mathbb{Z}\right)-\mathrm{H}_{1}(\alpha, \mathbb{Z})$ is an endomorphism of the free abelian group $\mathrm{H}_{1}\left(\Sigma_{2}, \mathbb{Z}\right)$, and its determinant is $\chi_{\mathbb{Z}}(\alpha)(t)$, which is $t^{4}-2 t^{3}+t^{2}-2 t+1$, by Lemma 2.2 . Clearly, $\chi_{\mathbb{Z}}(\alpha)(-1)=7$, so we see that the image of the endomorphism $\mathrm{H}_{1}\left(1_{\Sigma_{2}}, \mathbb{Z}\right)+\mathrm{H}_{1}(\alpha, \mathbb{Z})$ has index 7 in $\mathrm{H}_{1}\left(\Sigma_{2}, \mathbb{Z}\right)$.

Let us express the foregoing in terms of the group $G=\pi_{1}\left(\Sigma_{2}\right)$. Here we can identify $\mathrm{H}_{1}\left(\Sigma_{2}, \mathbb{Z}\right)=G / G^{\prime}$, where $G^{\prime}=\langle[x, y] \mid x, y \in G\rangle$, the derived subgroup of $G$. The image of $\mathrm{H}_{1}\left(1_{\Sigma_{2}}, \mathbb{Z}\right)+\mathrm{H}_{1}(\alpha, \mathbb{Z})$ is $H / G^{\prime}$, where $H=\left\langle[x, y], x \cdot \pi_{1}(\alpha)(x)\right| x, y \in$ $G\rangle$. Thus the previous paragraph shows that $H$ has index 7 in $G$. Since $H$ contains 
$G^{\prime}, H$ is a normal subgroup of $G$. Here $\pi_{1}(\alpha)$ maps $H$ to itself, and acts by inversion on $G / H$.

Consider the universal covering space $\tilde{\Sigma}_{2}$ of $\Sigma_{2}$. We have a free, properlydiscontinuous action of $G$ on $\tilde{\Sigma}_{2}$ by orientation-preserving self-homeomorphisms, and we can identify $G \backslash \tilde{\Sigma}_{2}=\Sigma_{2}$. Now $\alpha$ lifts to an orientation-preserving selfhomeomorphism $\tilde{\alpha}$ of $\tilde{\Sigma}_{2}$ in such a way that, for each $x \in G$, the action of $\tilde{\alpha} x \tilde{\alpha}^{-1}$ agrees with the action of $\pi_{1}(\alpha)(x)$.

Since $\pi_{1}(\alpha)$ maps $H$ to itself, $\tilde{\alpha}$ induces a well-defined orientation-preserving self-homeomorphism $\beta$ of the quotient space $H \backslash \tilde{\Sigma}_{2}$, and $G / H$ acts freely on $H \backslash \tilde{\Sigma}_{2}$ with quotient $G \backslash \tilde{\Sigma}_{2}=\Sigma_{2}$. The action of the group $G / H$ of order seven gives an orientation-preserving self-homeomorphism $\gamma$ of $H \backslash \tilde{\Sigma}_{2}$ such that $\gamma^{7}=1$. Since $\pi_{1}(\alpha)$ acts on $G / H$ by inversion, we see that $\beta \gamma \beta^{-1}=\gamma^{-1}$, that is, $\gamma \beta \gamma=\beta$.

Now $H \backslash \tilde{\Sigma}_{2} \rightarrow G \backslash \tilde{\Sigma}_{2}=\Sigma_{2}$ is a seven-fold regular covering, so $H \backslash \tilde{\Sigma}_{2}$ is a surface of genus eight, and we can write $\Sigma_{8}=H \backslash \tilde{\Sigma}_{2}$. The action induced on $\Sigma_{8}$ by $\tilde{\alpha}$, or equivalently by $\beta$, agrees with $\alpha$.

By Nielsen's equivalence theorem [9, Section 11] (cf. [7]), $\Sigma_{8}$ can be viewed as a torus with seven handles with $\gamma$ acting by rotating the torus, cyclically permuting the seven handles. The importance of this, for us, is that it implies that there exists a presentation

$$
G=\left\langle y_{1}, z_{1}, y_{2}, z_{2} \mid\left[y_{1}, z_{1}\right]\left[y_{2}, z_{2}\right]=1\right\rangle
$$

so that

$$
H=\left\langle w, x, w_{i}, x_{i}\left(i \in \mathbb{Z}_{7}\right) \mid[w, x] \prod_{i=0}^{6}\left[w_{i}, x_{i}\right]=1\right\rangle,
$$

where $w \mapsto y_{1}^{7}, x \mapsto z_{1}, w_{i} \mapsto y_{1}^{i} y_{2} y_{1}^{-i}, x_{i} \mapsto y_{1}^{i} z_{2} y_{1}^{-i}(0 \leq i \leq 6)$, and we can take $\pi_{1}(\gamma)$ to be left conjugation by $y_{1}$. (One can check this directly by showing that the $7^{4}-1$ surjective group homomorphisms from $G$ to $\mathbb{Z}_{7}$ are all the same, modulo composition with automorphisms of the domain and range. We leave the details to the reader who prefers not to invoke Nielsen's theorem.)

Let $R$ be as in Lemma 2.1, and set $M=\mathrm{H}_{1}\left(\Sigma_{8}, R\right), N=\mathrm{H}_{1}\left(\Sigma_{2}, R\right)$.

It is not difficult to show that, with $r$ as in Lemma $2.1, M$ has an $R$-basis as follows: $u=\frac{1}{7}[w], v=[x], u_{j}=\frac{1}{7} \sum_{i \in \mathbb{Z}_{7}} r^{i j}\left[w_{i}\right], v_{j}=\frac{1}{7} \sum_{i \in \mathbb{Z}_{7}} r^{i j}\left[x_{i}\right]\left(j \in \mathbb{Z}_{7}\right)$. For example, for any $k \in \mathbb{Z}_{7}$, we have

$$
\begin{aligned}
\sum_{j \in \mathbb{Z}_{7}} r^{k j} u_{j} & =\frac{1}{7} \sum_{j \in \mathbb{Z}_{7}} r^{k j} \sum_{i \in \mathbb{Z}_{7}} r^{i j}\left[w_{i}\right] \\
& =\frac{1}{7} \sum_{i \in \mathbb{Z}_{7}}\left(\sum_{j \in \mathbb{Z}_{7}}\left(r^{k+i}\right)^{j}\right)\left[w_{i}\right]=\frac{1}{7} \sum_{i \in \mathbb{Z}_{7}} 7 \delta_{i,-k}\left[w_{i}\right]=\left[w_{-k}\right] .
\end{aligned}
$$

Let $M_{0}$ denote the $R$-submodule of $M$ with $R$-basis $u, v, u_{0}, v_{0}$, and, for $1 \leq i \leq 6$, let $M_{i}$ be the $R$-submodule of $M$ with $R$-basis $u_{i}, v_{i}$. Then $M=\bigoplus_{i \in \mathbb{Z}_{7}} M_{i}$.

Let $A=\mathrm{H}_{1}(\alpha, R), B=\mathrm{H}_{1}(\beta, R)$, and $C=\mathrm{H}_{1}(\gamma, R)$. Then $C^{7}=1$ and $C B C=B$. Here $C$ acts by fixing $[w],[x]$ and cyclically permuting the $\left[w_{i}\right] \mathrm{s}$, and the $\left[x_{i}\right]$ s. Hence $C$ acts as multiplication by $r^{-i}$ on $M_{i}\left(i \in \mathbb{Z}_{7}\right)$.

Now consider the map $M \rightarrow N$ induced by our covering map $\Sigma_{8} \rightarrow \Sigma_{2}$. This forms a commuting diagram when combined with the endomorphisms $B$ and $A$. 
Here $[w] \mapsto 7\left[y_{1}\right],[x] \mapsto\left[z_{1}\right],\left[w_{i}\right] \mapsto\left[y_{2}\right],\left[x_{i}\right] \mapsto\left[z_{2}\right]\left(i \in \mathbb{Z}_{7}\right)$. Thus $u \mapsto\left[y_{1}\right]$, $v \mapsto\left[z_{1}\right], u_{j} \mapsto \frac{1}{7} \sum_{i \in \mathbb{Z}_{7}} r^{i j}\left[y_{2}\right]=\delta_{0, j}\left[y_{2}\right], v_{j} \mapsto \delta_{0, j}\left[z_{2}\right]\left(j \in \mathbb{Z}_{7}\right)$. Hence the map $M \rightarrow N$ carries $M_{0}$ bijectively to $N$, and has kernel Ker $=\bigoplus_{i=1}^{6} M_{i}$. We then have an identification $M_{0}=N$.

Let $i \in \mathbb{Z}_{7}$. Let us examine how $B$ acts on $M_{i}$. We know that

$$
0=(B-C B C) M_{i}=\left(B-C B r^{-i}\right) M_{i}=\left(B-r^{-i} C B\right) M_{i}=\left(I-r^{-i} C\right) B M_{i} .
$$

Hence $I-r^{-i} C$ vanishes on $B M_{i}$, so $B M_{i} \subseteq M_{-i}$. Let $B_{i}$ denote the induced map $M_{i} \rightarrow M_{-i}$. Since $B$ is an automorphism, $B_{i}$ is an isomorphism. Notice that $B^{2}$ carries $M_{i}$ to itself, acting as $B_{-i} B_{i}$.

In particular $B$ acts on $M_{0}$ as $B_{0}$. Recall that $B$ carries Ker to itself, and acts as $A$ on the quotient $M / \operatorname{Ker}=N$. As we now see that $B$ carries $M_{0}$ to itself, the identification $M_{0}=N$ allows us to identify $B_{0}=A$. Hence $\operatorname{trace}\left(B_{0}^{2}\right)=$ $\operatorname{trace}\left(A^{2}\right)=2$.

Let $j \in \mathbb{Z}_{7}$. If $\beta^{2} \gamma^{j}$ has a fixed-point $p$ in $\Sigma_{8}$, then the image of $p$ in $\gamma \backslash \Sigma_{8}=\Sigma_{2}$ is a fixed-point for the action induced by $\beta^{2}$, that is, for the action of $\alpha^{2}$. By our supposition, no such point exists, so $\beta^{2} \gamma^{j}$ is fixed-point free. By the Lefschetz fixed-point theorem, $\operatorname{trace}_{R}\left(\beta^{2} \gamma^{j}\right)=2$, that is, $\operatorname{trace}\left(B^{2} C^{j}\right)=2$. Hence

$$
\begin{aligned}
2 & =\operatorname{trace}\left(B^{2} C^{j}\right)=\operatorname{trace}\left(\bigoplus_{i \in \mathbb{Z}_{7}}\left(B^{2} C^{j}: M_{i} \rightarrow M_{i}\right)\right)=\sum_{i \in \mathbb{Z}_{7}} \operatorname{trace}\left(B_{-i} B_{i} r^{-i j}\right) \\
& =\sum_{i \in \mathbb{Z}_{7}} r^{-i j} \operatorname{trace}\left(B_{-i} B_{i}\right)=\operatorname{trace}\left(B_{0}^{2}\right)+\sum_{i=1}^{6} r^{-i j} \operatorname{trace}\left(B_{-i} B_{i}\right) \\
& =2+\sum_{i=1}^{6} r^{-i j} \operatorname{trace}\left(B_{-i} B_{i}\right) .
\end{aligned}
$$

That is, $\sum_{i=1}^{6} r^{-i j} \operatorname{trace}\left(B_{-i} B_{i}\right)=0$.

$$
\begin{aligned}
\operatorname{trace}\left(B_{-1} B_{1}\right) & =\sum_{i=1}^{6} \operatorname{trace}\left(B_{-i} B_{i}\right) \delta_{i, 1}=\sum_{i=1}^{6} \operatorname{trace}\left(B_{-i} B_{i}\right) \frac{1}{7} \sum_{j \in \mathbb{Z}_{7}}\left(r^{1-i}\right)^{j} \\
& =\frac{1}{7} \sum_{j \in \mathbb{Z}_{7}} r^{j}\left(\sum_{i=1}^{6} r^{-i j} \operatorname{trace}\left(B_{-i} B_{i}\right)\right)=\frac{1}{7} \sum_{j \in \mathbb{Z}_{7}} r^{j} 0=0 .
\end{aligned}
$$

Transposing, we have trace $\left(B_{1}^{t}\left(B_{-1}\right)^{t}\right)=0$.

Now consider the $R$-basis $u_{1}, v_{1}, 7 v_{-1},-7 u_{-1}$ of $M_{1} \oplus M_{-1}$. With respect to this basis the symplectic form induced from $M$ has the matrix $\left(\begin{array}{cc}0 & I_{2} \\ -I_{2} & 0\end{array}\right)$, since, for any $k, l \in \mathbb{Z}_{7}$, we have

$$
\begin{aligned}
\left\langle u_{k}, v_{l}\right\rangle & =\frac{1}{49} \sum_{i \in \mathbb{Z}_{7}} \sum_{j \in \mathbb{Z}_{7}} r^{i k} r^{j l}\left\langle\left[w_{i}\right],\left[x_{j}\right]\right\rangle=\frac{1}{49} \sum_{i \in \mathbb{Z}_{7}} \sum_{j \in \mathbb{Z}_{7}} r^{i k} r^{j l} \delta_{i, j} \\
& =\frac{1}{49} \sum_{i \in \mathbb{Z}_{7}} r^{i k} r^{i l}=\frac{1}{7} \delta_{k,-l} .
\end{aligned}
$$


With respect to the same $R$-basis, the action of the endomorphism $B$ on $M_{1} \oplus$ $M_{-1}$ can be viewed as having the form $\left(\begin{array}{cc}0 & B_{-1} \\ B_{1} & 0\end{array}\right)$. The fact that $B$ respects the symplectic form implies that $\left(\begin{array}{cc}0 & B_{-1} \\ B_{1} & 0\end{array}\right)^{t}\left(\begin{array}{cc}0 & I_{2} \\ -I_{2} & 0\end{array}\right)\left(\begin{array}{cc}0 & B_{-1} \\ B_{1} & 0\end{array}\right)=\left(\begin{array}{cc}0 & I_{2} \\ -I_{2} & 0\end{array}\right)$, that is,

$$
\left(\begin{array}{cc}
0 & -\left(B_{1}\right)^{t} B_{-1} \\
\left(B_{-1}\right)^{t} B_{1} & 0
\end{array}\right)=\left(\begin{array}{cc}
0 & I_{2} \\
-I_{2} & 0
\end{array}\right),
$$

so $\left(B_{-1}\right)^{t} B_{1}=-I_{2}$, and thus $B_{1}^{-1}=-\left(B_{-1}\right)^{t}$.

Combining the results of the previous two paragraphs we have

$$
\operatorname{trace}\left(B_{1}^{t} B_{1}^{-1}\right)=-\operatorname{trace}\left(B_{1}^{t}\left(B_{-1}\right)^{t}\right)=0,
$$

which contradicts the property of $R$ given in Lemma 2.1.

\section{An exAmple}

In proving Theorem 2.3 above, we are showing that if $\alpha$ is an orientationpreserving self-homeomorphism of $\Sigma_{2}$ such that $\chi_{\mathbb{Z}}(\alpha)(t)=t^{4}-2 t^{3}+t^{2}-2 t+1$, then $\alpha^{2}$ has a fixed-point. Hence the question arises whether such $\alpha$ exist, and the answer is that they do. Boju Jiang pointed out to us that this must have been known to Nielsen, since examples can be obtained by taking the group automorphism constructed in Example 13, Section 49 of Nielsen's seminal paper [8] (cf. $[6]$ ), and composing it with any group automorphism inducing negation on the first homology group. The purpose of this section is to describe one example in detail.

Let us give $\Sigma_{2}$ a CW-structure as follows. Take two vertices, $p_{1}, p_{2}$ and five edges $e_{i}, 1 \leq i \leq 5$, joining $p_{1}$ to $p_{2}$, oriented so that the "odd" edges start at $p_{1}$ and the "even" edges start at $p_{2}$. Adjoining an oriented decagonal two-cell $F$ with boundary $\partial F=e_{1} e_{2} e_{3} e_{4} e_{5} e_{1}^{-1} e_{2}^{-1} e_{3}^{-1} e_{4}^{-1} e_{5}^{-1}$ gives a $\mathrm{CW}$-structure on $\Sigma_{2}$; see Figure $1(\mathrm{a})$.

We now define two different subdivisions, as in Figures 1(b), (c). Thus we decompose $F$ into an oriented edge denoted $e_{0}$ (resp. $\left.e_{6}\right)$ joining $p_{2}$ to $p_{1}$, and two oriented hexagonal two-cells denoted $F_{1}, F_{2}$ (resp. $\left.\alpha\left(F_{1}\right), \alpha\left(F_{2}\right)\right)$ with $\partial F_{1}=$ $e_{1} e_{2} e_{3} e_{4} e_{0}^{-1} e_{5}^{-1}, \partial F_{2}=e_{0} e_{5} e_{1}^{-1} e_{2}^{-1} e_{3}^{-1} e_{4}^{-1}$ (resp. $\partial \alpha\left(F_{1}\right)=e_{2} e_{3} e_{4} e_{5} e_{1}^{-1} e_{6}^{-1}, \partial \alpha\left(F_{2}\right)=$ $\left.e_{1} e_{6} e_{2}^{-1} e_{3}^{-1} e_{4}^{-1} e_{5}^{-1}\right)$.

Now take a CW-map, from the CW-complex in Figure 1(b) to the CW-complex in Figure $1(\mathrm{c})$, which sends $p_{1}$ to $p_{2}, p_{2}$ to $p_{1}, e_{i}$ to $e_{i+1}(0 \leq i \leq 5), F_{1}$ to $\alpha\left(F_{1}\right)$ and $F_{2}$ to $\alpha\left(F_{2}\right)$. At the level of underlying spaces, this defines an orientation-preserving self-homeomorphism $\alpha: \Sigma_{2} \rightarrow \Sigma_{2}$.

Here $\pi_{1}\left(\Sigma_{2}, p_{1}\right)$ is generated by the homotopy classes of paths $a=\left[e_{1} e_{2}\right], b=$ $\left[e_{3} e_{1}^{-1}\right], c=\left[e_{1} e_{4}\right], d=\left[e_{5} e_{1}^{-1}\right]$, and the defining relation is $a b c d a^{-1} b^{-1} c^{-1} d^{-1}=1$, where square brackets denote homotopy classes.

To define $\pi_{1}(\alpha)$, it remains to choose a homotopy class of paths from $p_{1}$ to $\alpha\left(p_{1}\right)=p_{2}$, and we choose $\left[e_{1}\right]$. Since $\left[e_{6}\right]=\left[e_{1}^{-1} e_{5} e_{4} e_{3} e_{2}\right]$, we see that $\pi_{1}(\alpha)$ is then the automorphism of $\pi_{1}\left(\Sigma_{2}\right)$ given by $(a, b, c, d) \mapsto\left(a b, c a^{-1}, a d, d c b\right)$. A straightforward calculation now shows that $\chi_{\mathbb{Z}}(\alpha)(t)=t^{4}-2 t^{3}+t^{2}-2 t+1$, as desired.

It is easy to check that there is a unique largest quotient group of $\pi_{1}\left(\Sigma_{2}\right)$ on which $\pi_{1}(\alpha)$ acts by inversion; it has order seven. One can then construct a $\beta$ and 

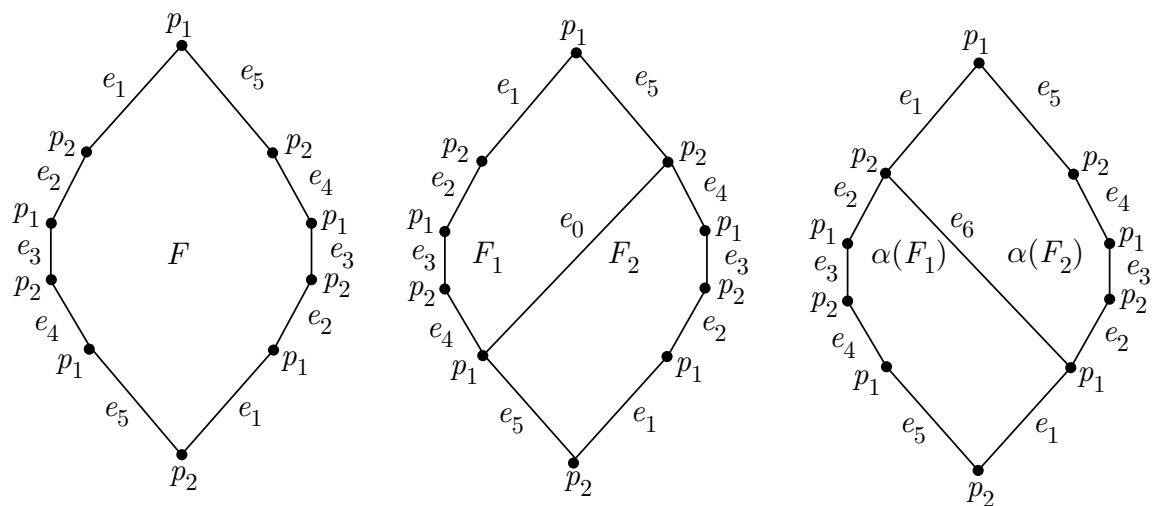

FiguRE 1

a $\gamma$ as in the proof of Theorem 2.3, and find that three of the $\beta^{2} \gamma^{j}, j \in \mathbb{Z}_{7}$, have trace 2 , while four of them have trace -5 , so have fixed-points. It follows that $\alpha^{2}$ has at least four homotopy fixed-points.

One can estimate the number of homotopy fixed points of $\alpha, \alpha^{2}$ algebraically, using results of Fadell and Husseini [5, Section 2]; in fact, it was their abelianization technique that suggested to us the idea of taking $t=-1$, which is the starting point of our proof of Theorem 2.3. Applying the formula in [5, Theorem 2.3], one finds, without too much difficulty, that $\alpha$ has no homotopy fixed-points, and that the abelianization technique shows that $\alpha^{2}$ has exactly four homotopy fixed-points, two of index +1 and two of index -1 . Hence the sum of the indices is 0 , which is why the Lefschetz fixed-point theorem cannot detect the existence of any fixedpoints for $\alpha^{2}$. For details on the theory of indices of isolated fixed-points, see, for example, [3].

We will now give a geometric verification of this result, by exhibiting a pseudoAnosov realization of $\alpha$ and calculating the points of period one or two. To obtain this realization we applied the elegant algorithm of Bestvina and Handel [2] which, given a surface homeomorphism, decides if it is equivalent to a pseudo-Anosov homeomorphism and, in the event that it is, yields a rectangular decomposition for an equivalent pseudo-Anosov homeomorphism.

Let $\lambda>\mu$ denote the the two real roots of our polynomial, $t^{4}-2 t^{3}+t^{2}-2 t+1$, so we have $\lambda=\frac{1+\sqrt{2}+\sqrt{-1+2 \sqrt{2}}}{2}=1.8832 \ldots$ and $\mu=\lambda^{-1}=\frac{1+\sqrt{2}-\sqrt{-1+2 \sqrt{2}}}{2}=$ $0.531 \ldots$. In order to simplify the formulae in the discussion that follows, whenever we reduce polynomial expressions in $\lambda$ and $\mu$, we shall use the fact that they are roots of

$$
t^{6}-2 t^{5}+2 t-1=\left(t^{2}-1\right)\left(t^{4}-2 t^{3}+t^{2}-2 t+1\right),
$$

rather than using our standard polynomial.

Let $D$ denote the decagon in the Euclidean plane with counter-clockwise vertex sequence

$$
\begin{aligned}
& (1,1),(\lambda, \mu),\left(\lambda^{2}, \mu^{2}\right),\left(\lambda^{3}, \mu^{3}\right),\left(\lambda^{4}, \mu^{4}\right),\left(\lambda^{5}, \mu^{5}\right), \\
& \left(\lambda^{5}-\lambda+1, \mu^{5}-\mu+1\right),\left(\lambda^{5}-\lambda^{2}+1, \mu^{5}-\mu^{2}+1\right), \\
& \left(\lambda^{5}-\lambda^{3}+1, \mu^{5}-\mu^{3}+1\right),\left(\lambda^{5}-\lambda^{4}+1, \mu^{5}-\mu^{4}+1\right),
\end{aligned}
$$




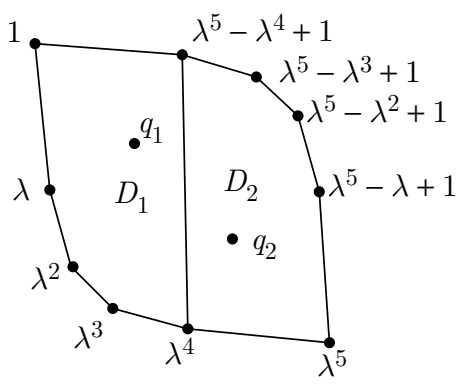

$D$

Figure 2

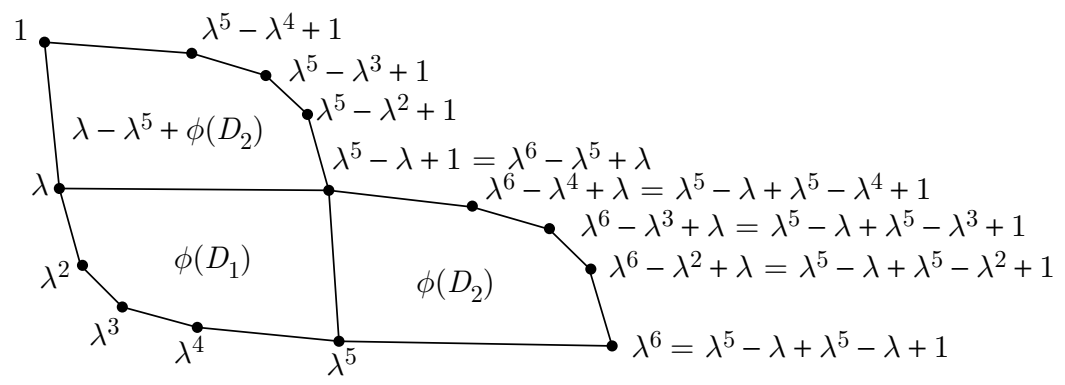

FiguRE 3

and denote by $D_{1}$ (resp. $D_{2}$ ) the left (resp. right) of the two hexagonal regions into which $D$ is divided by the straight line segment joining the fifth point to the tenth point; see Figure 2, where the vertices are labelled with their $x$-coordinates and, for aesthetic reasons, we have used a scale in which one unit in the $y$ direction equals $\lambda^{5}$ units in the $x$ direction.

Pairs of opposite sides of $D$ are parallel, and we identify them, via a Euclidean translation, to form $\Sigma_{2}$, which then has a co-ordinate system. We denote the map $D \rightarrow \Sigma_{2}$ by $(x, y) \mapsto[(x, y)]$.

The linear map $\phi: \mathbb{R}^{2} \rightarrow \mathbb{R}^{2}$ defined by $(x, y) \mapsto(\lambda x, \mu y)$ transforms $D$ as depicted in Figure 3, and hence determines a discontinuous piecewise-linear map on $D$, which translates $\phi(D)-D\left(=\phi\left(D-D_{1}\right)\right)$ back to $D$. The map on $D$ behaves well with respect to the edge identifications and determines a self-homeomorphism, denoted $\alpha$, on $\Sigma_{2}$. Thus, for any $(x, y) \in D$,

$$
\alpha[(x, y)]= \begin{cases}{[(\lambda x, \mu y)]} & \text { if }(x, y) \in D_{1}, \\ {\left[\left(\lambda-\lambda^{5}+\lambda x, \mu-\mu^{5}+\mu y\right)\right]} & \text { if }(x, y) \in D_{2} .\end{cases}
$$

On comparing Figures 1(b) and (c) with Figures 2 and 3, we see that we have a geometric realization of the map which was constructed above in terms of CWcomplexes.

In $\Sigma_{2}$, let

$$
\begin{gathered}
p_{1}=[(1,1)], \quad p_{2}=[(\lambda, \mu)], \\
q_{1}=\left[\left(\lambda+\lambda^{3}, \mu+\mu^{3}\right)\right], \quad q_{2}=\left[\left(\lambda^{2}+\lambda^{4}, \mu^{2}+\mu^{4}\right)\right] .
\end{gathered}
$$


It is straightforward to check that $\alpha$ is fixed-point free, interchanges $p_{1}, p_{2}$, interchanges $q_{1}, q_{2}$, and has no other points of period two. Thus the fixed-points of $\alpha^{2}$ are $p_{1}, p_{2}, q_{1}, q_{2}$. The index of $p_{1}$ and $p_{2}$ is +1 , since, near them, $\alpha^{2}$ behaves like rotation through an angle of $-\pi$. Similarly, the index of $q_{1}$ and $q_{2}$ is -1 , since, near them, $\alpha^{2}$ behaves like $(x, y) \mapsto\left(\lambda^{2} x, \mu^{2} y\right)$. For details on calculating indices of isolated fixed-points of this type, see, for example, [1].

The horizontal and vertical Euclidean lines in $D$, with the usual Lebesgue measure, determine transverse measured foliations on $\Sigma_{2}$, preserved by $\alpha$; the action of $\alpha$ expands (resp. contracts) the measured foliation determined by the horizontal (resp. vertical) lines, by a factor of $\lambda$ (resp. $\mu=\lambda^{-1}$ ). The only singularities are at $p_{1}$ and $p_{2}$, and they are 4-prong singularities. This means that $\alpha$ is pseudo-Anosov.

\section{ACKNOWLEDGMENTS}

We thank Mladen Bestvina, Henry Glover, John Guaschi, Mike Handel, Boju Jiang and Jorge Morales for various useful pieces of information fundamental to our eventual understanding of the problem.

We are grateful to the DGICYT for generous support through grants PB93-0900 and PB93-0860.

\section{REFERENCES}

1. P. Alexandroff and H. Hopf, Topologie, Springer-Verlag, Berlin, 1974. MR 49:9826

2. M. Bestvina and M. Handel, Train tracks for surface homeomorphisms, Topology 34 (1995), 109-140.

3. R.F. Brown, The Lefschetz fixed point theorem, Scott, Foresman and Co., Glenview, 1971. MR 44:1023

4. B. Doubrovine, S. Novikov and A. Fomenko, Géométrie Contemporaine, $3^{e}$ partie de Méthodes de la théorie de l'homolgie, Editions Mir, Moscow, 1987.

5. E.R. Fadell and S. Husseini, The Nielsen numbers on surfaces, Topological methods in nonlinear functional analysis (S.P. Singh, S. Thomeier, and B. Watson, eds.), Contemporary Mathematics, vol. 21, Amer. Math. Soc., Providence, RI, 1983, pp. 59-98. MR 85h:55004

6. V.L. Hansen (editor), Jakob Nielsen, collected mathematical papers, Volume 1 (1913-1932), Birkhäuser, Basel, 1986. MR 88a:01070a

7. V.L. Hansen (editor), Jakob Nielsen, collected mathematical papers, Volume 2 (1932-1955), Birkhäuser, Basel, 1986. MR 88a:01070b

8. J. Nielsen, Untersuchungen zur Topologie der geschlossenen zweiseitigen Flächen, Acta Math. 50 (1927), 189-358.

9. J. Nielsen, Die Struktur periodischer Transformationen von Flächen, Math.-fys. Medd. Denske Vid. Selsk. 15 (1937), 1-77.

10. J. Nielsen, Fixpunktfrie Afbildninger, Mat. Tidsskr. B (1942), 25-41. MR 7:137c

11. C.L. Siegel, Symplectic geometry, Academic Press, New York, 1964. MR 29:1362

12. E.H. Spanier, Algebraic topology, McGraw-Hill, New York, 1966. MR 35:1007

13. S. Wang, Free degrees of homeomorphisms and periodic maps on closed surfaces, Topology Appl. 46 (1992), 81-87. MR 93g:55003

Departament de Matemàtiques, Universitat Autònoma de Barcelona,08193 Bellaterra (Barcelona), Spain

E-mail address: dicks@mat.uab.es

E-mail address: imat0@cc.uab.es 